\title{
Hedging a Portfolio of Derivatives by Modeling Cost Cot $^{*+}$
}

\author{
Katharyn A. Boyle**, Thomas F. Coleman, and Yuying Li \\ Department of Computer Science, Cornell University, Ithaca, New York, USA \\ ${ }^{* *}$ Center for Applied Mathematics, Cornell University, Ithaca, New York, USA \\ Email: katharyn@cam.cornell.edu ,coleman@tc.cornell.edu, yuying@tc.cornell.edu
}

Jan 13, 2003

\begin{abstract}
We consider the problem of hedging the loss of a given portfolio of derivatives using a set of more liquid derivative instruments. We illustrate why the typical mathematical formulation for this hedging problem is ill-posed. We propose to determine a hedging portfolio by minimizing a proportional cost subject to an upper bound on the hedge risk; this bound is typically slightly larger than the optimal hedge risk achievable without cost consideration. We illustrate that the optimal hedging portfolio obtained by the proposed method is attractive since it consists of fewer instruments with a comparable risk. Finally we illustrate the importance of modeling volatility uncertainty in hedge risk minimization.
\end{abstract}

Keywords. hedging, risk minimization, Black-Scholes formula, ill-posedness, stochastic volatility

\section{Introduction}

Measuring and improving risk is important in risk management and it is of great interest to both practioners as well as academics. Hedging is a key approach to reduce the risk of a single instrument as well as a portfolio of derivatives. Carr and $\mathrm{Wu}$ [2] recently compare the dynamic delta hedging with a static hedging of a single European option of a long term maturity, using European options of different strikes of a shorter maturity. In this paper, we focus on hedging a portfolio of a large number of derivatives with more liquid derivative instruments.

In practice, hedging a portfolio of derivatives is typically done through matching of different sensitivities between the given portfolio and the hedging portfolio. As an alternative, a hedging portfolio can be chosen to minimize a measure of the hedge risk for a given time horizon, e.g., the expected quadratic difference between the future loss of the target portfolio and the value of the hedging portfolio.

A potential problem with both sensitivity matching and hedge risk minimization is that it can lead to a hedging portfolio consisting of a large number of instruments as well as large units of instrument holdings, as illustrated in [4]. Assume, for example, that we want to hedge a short term at-the-money call with a deep in-the-money midterm call and the underlying. The result from matching the delta and gamma sensitivities yields a hedging portfolio of a large long position of the deep in-the-money call and a large short position of the underlying asset. The risk minimization approach encounters a similar problem; it essentially searches for the optimal replicating portfolio that matches the future values in distribution. Assume that the underlying has a lognormal distribution. The distribution of the future values of the at-the-money option is far from lognormal while the distribution of the deep in-the-money midterm maturity call is closer to lognormal.

\footnotetext{
${ }^{*}$ This research was conducted using resources of the Cornell Theory Center, which is supported in part by Cornell University, New York State, and members of the Corporate Partnership Program.

${ }^{\dagger}$ To appear: IEEE Proceedings of the 2003 International Conference on Computational Intelligence for Financial Engineering (CIFEr2003), March 21-23, 2003, Hong Kong.
} 
Thus the hedge risk minimization model also leads to a portfolio of large long and short holdings of the in-the-money call and the underlying respectively. This problem is generic when hedging a portfolio of a large number of derivative instruments and underlyings [4].

Tompaidis [4] proposes to overcome the above difficulty by identifying a subset of the most important instruments in hedging the loss of a target portfolio by adding hedging instruments sequentially to reduce the hedge risk; the iterative search process is terminated when the hedge risk is no longer significantly improved with the addition of a new instrument.

In this paper, we illustrate that the difficulty in hedging the portfolio loss through sensitivity matching and hedge risk minimization often arises from the fact that the total number of risk factors in the assumed model for the underlying of the hedging instruments is small relative to the total number of hedging instruments. We propose a risk minimization model to account for hedging cost, transaction cost, and management cost; a hedging portfolio is determined by minimizing a proportional cost subject to an upper bound on the hedge risk which is typically slightly larger than the optimal hedge risk achievable without cost consideration. The relaxation of the hedge risk is reasonable and prudent in light of the inevitable model error. The proposed hedge risk minimization model produces a more desirable hedging strategy; the optimal hedging portfolio incurs a smaller cost and often has a smaller number of instruments. Finally, we illustrate why modeling volatility uncertainty in hedge risk minimization is important even though the exact distribution for the future (implied) volatility is unknowable.

\section{An Ill-posed Problem}

Suppose that we have a portfolio of vanilla and exotic options on a single stock; the exotic options include binary, barrier, and Asian options. We want to hedge the potential future loss of the existing portfolio using the underlying stock and a set of liquid vanilla options on the same stock. For example, the hedging instruments are the underlying stock, at-the-money and near the money call options with maturity one, two, three and six months. Which portfolio is best in hedging against the future loss of our existing portfolio?

To form this problem mathematically, let us assume more generally that the underlying risk factors $S \in \Re^{d}$ of the hedging instruments $\left\{V_{1}, V_{2}, \cdots, V_{n}\right\}$ is a square integrable process on a probability space $\left(\Omega, \mathcal{F}_{t}, P\right)$ with a filtration $\mathcal{F}_{t}$. Let $V_{i}(S, t)$ denote a derivative instrument value at time $t$ when underlying risk is $S$. Let $\Pi(x ; S, t) \stackrel{\text { def }}{=} \sum_{i=1}^{n} x_{i} V_{i}(S, t)$ denote the value of the hedging portfolio at time $t$ and $\Pi^{0}(S, t)$ denote the loss of the target portfolio at time $t$. Given a hedge horizon $\tilde{t}>0$, the loss $\Pi^{0}$ is the initial value of the target portfolio minus the time $\tilde{t}$ value of the target portfolio. We want to determine a portfolio $x$ so that a measure of the hedge risk is minimized when the portfolio $\Pi(x ; S, \tilde{t})$ is used to hedge the loss $\Pi^{0}(S, \tilde{t})$.

Let the row vector $V \stackrel{\text { def }}{=}\left[V_{1}, \cdots, V_{n}\right]$ denote the hedging instrument values at $t>0(\Pi(x ; S, t)=$ $\left.\sum_{i=1}^{n} x_{i} V_{i}(S, t)=V x\right)$ with their sensitivities as below:

$$
\begin{aligned}
& \frac{\partial V}{\partial t} \stackrel{\text { def }}{=}\left[\frac{\partial V_{1}}{\partial t}, \cdots, \frac{\partial V_{n}}{\partial t}\right] \in \Re^{1 \times n} \\
& \frac{\partial V}{\partial S} \stackrel{\text { def }}{=}\left[\frac{\partial V_{1}}{\partial S}, \cdots, \frac{\partial V_{n}}{\partial S}\right] \in \Re^{d \times n} \\
& \Gamma \stackrel{\text { def }}{=}\left[\Gamma_{1}, \cdots, \Gamma_{n}\right] \in \Re^{d \times n}
\end{aligned}
$$

where the column vector $\frac{\partial V_{i}}{\partial S} \stackrel{\text { def }}{=}\left[\frac{\partial V_{i}}{\partial S_{1}}, \cdots, \frac{\partial V_{i}}{\partial S_{d}}\right]^{T}$ and and $\Gamma_{i} \stackrel{\text { def }}{=}\left[\frac{\partial^{2} V_{i}}{\partial S_{1}^{2}}, \cdots, \frac{\partial^{2} V_{i}}{\partial S_{d}^{2}}\right]^{T}$. For simplicity of presentation, we assume here that each hedging instrument $V_{i}$ depends on at most one risk factor.

Assume that one is to hedge the loss $\Pi^{0}$ of a derivative portfolio by matching delta, gamma, and theta 
sensitivities. To accomplish this, solve

$$
\left[\begin{array}{c}
\frac{\partial V}{\partial t} \\
\frac{\partial V}{\partial S} \\
\Gamma
\end{array}\right] x=\left[\begin{array}{c}
\frac{\partial \Pi^{0}}{\partial t} \\
\frac{\partial \Pi^{0}}{\partial S} \\
\Gamma_{\Pi^{0}}
\end{array}\right]
$$

where the column vector $\Gamma_{\Pi^{0}} \stackrel{\text { def }}{=}\left[\frac{\partial^{2} \Pi^{0}}{\partial S_{1}^{2}}, \cdots, \frac{\partial^{2} \Pi^{0}}{\partial S_{n}^{2}}\right]^{T}$ represents gamma sensitivities of the loss of the target portfolio. Note that the coefficient matrix has dimension $(2 d+1) \times n$. If $n>2 d+1$, the linear system (1) is underdetermined. Hence there are an infinite number of portfolios matching these sensitivities. It is difficult to determine which $2 d+1$ hedging instruments to use to best hedge a given portfolio; a portfolio with large positions can occur if hedging instruments are not chosen appropriately. For example, if a short term at-the-money call is matched in sensitivity by the underlying and a midterm at-the-money call, the hedging instrument positions are much smaller, compared to using the underlying and a midterm deep-inthe money call considered previously. In addition, matching sensitivities to control hedging risk is limited in its effectiveness unless the hedge horizon is sufficiently small.

Minimizing a measure of the hedge risk is an alternative to sensitivity matching. In this paper, we measure the hedge risk as the expected quadratic replicating error; hence the minimization problem is

$$
\min _{x \in \Re^{n}} \operatorname{risk}(x) \stackrel{\text { def }}{=} \mathbf{E}\left(\left(\sum_{i=1}^{n} x_{i} V_{i}(S, t)-\Pi^{0}(S, t)\right)^{2}\right)
$$

where $\mathbf{E}$ denote the expectation with respect to a probability measure $P$ conditional on $S=S$ at $t=0$. The optimal hedging portfolio depends on the chosen risk measure; a comparison of hedging cost and risk between the choice of quadratic and piecewise risk measures, for discrete hedging a single vanilla instrument with the underlying and bond, can be found in [3].

To analyze the conditioning of the risk minimization formulation (2), let us assume for now that the change in the hedging instrument value is specified by the delta-gamma approximation:

$$
\begin{aligned}
& V_{i}(S, t)-V_{i}\left(S_{0}, 0\right) \\
= & \left(\frac{\partial V_{i}}{\partial t}\right) \delta t+\left(\frac{\partial V_{i}}{\partial S}\right)^{T}(\delta S)+\frac{1}{2}(\delta S)^{T}\left(\frac{\partial^{2} V_{i}}{\partial S^{2}}\right)(\delta S) \\
= & \left(\frac{\partial V_{i}}{\partial t}\right) \delta t+\left(\frac{\partial V_{i}}{\partial S}\right)^{T}(\delta S)+\frac{1}{2}\left(\Gamma_{i}\right)^{T}(\delta S)^{2} .
\end{aligned}
$$

Here the column vector $\delta S \in \Re^{d},(\delta S)^{2} \in \Re^{d}$ is the vector with each component of $\delta S$ squared, and $\frac{\partial^{2} V_{i}}{\partial S^{2}}$ is the $d$-by- $d$ Hessian matrix. Note the assumption that each hedging instrument $V_{i}$ depends on at most one risk factor is used to obtain the last equality above.

We show next that, if $n>2 d+1$ and (3) holds, then there are an infinite number of portfolios which give the same hedge risk. To see this, we first note that if portfolios $x^{(1)}, x^{(2)} \in \Re^{n}$ satisfy

$$
\Pi\left(x^{(1)} ; S, t\right) \equiv \Pi\left(x^{(2)} ; S, t\right), \quad \text { for all possible } S,
$$

then these two portfolios have the same hedge risk at time $t$.

Let $A^{T} \in \Re^{n \times(2 d+1)}$ denote the matrix below:

$$
A^{T} \stackrel{\text { def }}{=}\left[V\left(S_{0}, 0\right)^{T}+\left(\frac{\partial V}{\partial t}\right)^{T} \delta t,\left(\frac{\partial V}{\partial S}\right)^{T}, \frac{1}{2} \Gamma^{T}\right]
$$

The proof is straightforward from the observation that, under the assumption (3),

$$
\Pi(x ; S, t)=V x=x^{T} A^{T}\left[\begin{array}{c}
1 \\
\delta S \\
(\delta S)^{2}
\end{array}\right]
$$


Assuming $n>2 d+1$, there exists a nonzero vector $z \in \Re^{n}$ satisfying $A z=0$. Then $\Pi(x+\alpha v ; S, t)=$ $\Pi(x ; S, t)$ for any $S$ and $\alpha$.

Note that if a hedging instrument $V_{i}$ depends on more than one risk factor a similar analysis can be done with relevant cross partial derivative terms accounted for; it can be shown that the solution to (2) is not unique when

$$
n>\left(1+d+\sum_{i=1}^{d} \sum_{i \leq j \leq d} I_{i j}\right)
$$

where $I$ is a $d$-by- $d$ indicator matrix with $I_{i j}=1$ if $\sum_{k=1}^{n}\left|\frac{\partial^{2} V_{k}}{\partial S_{i} \partial S_{j}}\right|>0$ and $I_{i j}=0$ otherwise. Note that, the inequality (5) typically holds in practice since the number of hedging instrument $n$ is usually much larger than the number of risk factors $d$ and each hedging instrument value typically depends only on a small number of risk factors. In [1], the ill-posedness of VaR and CVaR minimization for a derivative portfolio is similarly analyzed.

When the derivative values are not computed by delta-gamma approximation, hedging by minimizing the hedge risk measure remains ill-posed. To illustrate, let us consider the single underlying asset derivative portfolio example described in the beginning of this section. Assume that the stock price $S$ follows a geometric Brownian motion

$$
\frac{d S_{t}}{S_{t}}=\mu d t+\sigma d X_{t}
$$

where $\mu>0$ is the expected rate of return, $\sigma>0$ is the volatility, and $X_{t}$ is a standard Brownian motion. Let the initial stock price $S_{0}=100$, the implied volatility $\sigma=0.2$, the expected rate of return $\mu=0.1$, and the risk free interest rate $r=0.04$. For simplicity, we assume that the stock pays no dividend. Assume further that the hedging instrument universe consists of the stock, and vanilla calls with the maturities of one, two, three, and six months and strikes $K=[90,95,100,105,110]$.

Let $S^{i}, i=1, \cdots, m$, be independent samples of the underlying stock price according to (6). The continuous minimization (2) is approximated by:

$$
\min _{x \in \Re^{n}} \operatorname{risk}(x)=\frac{1}{m} \sum_{i=1}^{m}\|\mathbf{V} x-b\|_{2}^{2}
$$

where $\mathbf{V}$ is defined as

$$
\left[\begin{array}{cccc}
V_{1}\left(S^{1}, t\right) & V_{2}\left(S^{1}, t\right) & \cdots & V_{n}\left(S^{1}, t\right) \\
\vdots & \vdots & \cdots & \vdots \\
V_{1}\left(S^{m}, t\right) & V_{2}\left(S^{m}, t\right) & \cdots & V_{n}\left(S^{m}, t\right)
\end{array}\right]
$$

and

$$
b \stackrel{\text { def }}{=}\left[\begin{array}{c}
\Pi^{0}\left(S^{1}, t\right) \\
\vdots \\
\Pi^{0}\left(S^{m}, t\right)
\end{array}\right]
$$

and $V_{j}\left(S^{i}, \tilde{t}\right)$ and $\Pi^{0}\left(S^{i}, \tilde{t}\right)$ are the $j^{t h}$ hedging instrument value and the loss of the given portfolio under scenario $i$ respectively.

The ill-posedness of problem (7) is reflected by the condition number of the matrix $V$. For our example, the condition number of the matrix $\mathbf{V}$ is of order $10^{16}$ for a short hedge horizon up to a month (computed using $m=20000$ independent price samples $S_{\tilde{t}}$ based on (6) with the parameters specified). The condition of $\mathbf{V}$ improves slightly as $\tilde{t}$ increases (the delta-gamma approximation becomes less accurate).

To examine the properties of the optimal hedging portfolio from the risk minimization (7), we consider four randomly generated target portfolios: a portfolio $P_{\mathrm{V}}$ of vanilla options, a portfolio $P_{\mathrm{bi}}$ of binary options, 
Table 1: Properties of the Optimal Portfolios

\begin{tabular}{|l|c|c|c|c|}
\hline & $P_{\mathrm{V}}$ & $P_{\mathrm{bi}}$ & $P_{\mathrm{ba}}$ & $P_{\mathrm{m}}$ \\
\hline$\sqrt{\text { risk }^{*}}$ & $7.27 \mathrm{e}-4$ & $3.05 \mathrm{e}-3$ & $8.04 \mathrm{e}-3$ & $7.05 \mathrm{e}-2$ \\
\hline$\left\|x^{*}\right\|_{1}$ & $1.93 \mathrm{e}+6$ & $3.08 \mathrm{e}+6$ & $7.47 \mathrm{e}+7$ & $1.87 \mathrm{e}+7$ \\
\hline
\end{tabular}

a portfolio $P_{\mathrm{ba}}$ of barrier options, and a portfolio $P_{\mathrm{m}}$, consisting of a mix of the three options plus Asian options. The strikes and maturities of the options in each target portfolios are $K=(50,60, \cdots, 150)$ and $T=(.1,0.2, \cdots, 1) \times 9 / 12$ with the positions (a $60 \%$ probability of being long and $40 \%$ probability of being short) of the options sampled from a lognormal distribution.

Table 1 displays the risk, as defined by (7), and the total number of positions of the optimal portfolios. Here $\left\|x^{*}\right\|_{1}=\sum_{i}\left|x_{i}^{*}\right|$ is the total number of instrument positions in a portfolio. Firstly, we note that all the optimal portfolios have impractically large positions with the total number of hedging position $\left\|x^{*}\right\|_{1}$ of a portfolio up to $O\left(10^{7}\right)$. Secondly, under the assumption that the implied volatility at $\tilde{t}$ is the same as that at $t=0$, optimal portfolios produced by the risk minimization model (7) has a relatively small risk.

\section{Hedging Under Cost}

In the last section, we have shown that the hedge risk minimization (2) typically produces a hedging portfolio with unrealistically large positions. This requires us to re-examine what characteristics we desire from the hedging portfolio. The ill-posedness of the hedge risk minimization model (2) indicates that risk minimization by itself is an insufficient criterion for choosing a hedging portfolio; there are many portfolios having the same or similar hedge risk. We discuss a few additional properties that can be introduced in the risk minimization to obtain a more attractive hedging portfolio.

Firstly, to avoid a hedging portfolio with unrealistically large positions, simple bound constraints can easily be included in the risk minimization formulation:

$$
\begin{array}{ll} 
& \min _{x \in \Re^{n}} \operatorname{risk}(x) \\
\text { subject to } & l_{x} \leq x \leq u_{x} .
\end{array}
$$

Here $l_{x}$ and $u_{x}$ denote the lower and upper bounds on the units of the positions for the instruments respectively.

Secondly, in addition to risk, cost is another important factor to consider. This cost usually includes the cost of forming the portfolio initially, transaction cost, as well as the portfolio management cost. Note that bounds on the initial cost of forming a hedging portfolio can be specified by $k \leq V^{0} x \leq u_{c}$ where $V^{0}=\left[V_{1}\left(S_{0}, 0\right), V_{2}\left(S_{0}, 0\right), \cdots, V_{n}\left(S_{0}, 0\right)\right], l_{c}$ and $u_{c}$ denote the lower and upper bounds on the initial cost. In this paper, we have not imposed any bound on the initial cost in order to analyze the minimal risk achievable.

The cost for management of a portfolio is much harder to model. It is reasonable to regard the management cost of a portfolio as proportional to the total number of distinct instruments in the portfolio, which leads to the problem

$$
\begin{array}{ll} 
& \min _{x \in \Re^{n}} \operatorname{risk}(x)+\alpha \sum_{i=1}^{n}\left[x_{i}\right]_{0} \\
\text { subject to } & l_{x} \leq x \leq u_{x}
\end{array}
$$


where $\left[x_{i}\right]_{0}=1$ if $x_{i} \neq 0$ and 0 otherwise. Another (equivalent) model accounting for management cost is the following: minimize the hedge risk using $n_{0}$ instruments among a universe of $n$ instruments, with $n_{0}<n$ a given integer. This is similar to the index tracking portfolio problem in equity portfolio management. It is an instance of portfolio compression problem and can potentially have many applications in risk management.

Unfortunately, solving (11) or minimizing risk using a fixed number of instruments in a given universe, is a very challenging computational problem. Tompaidis proposes [4] a matching projection pursuit method to identify a subset of the most important instruments. It can be shown that, for the first $n$ iterations, the matching projection pursuit algorithm is simply an application of the Gauss-Seidel method to the normal equations with a specific order for updating. Although the matching projection pursuit algorithm may be appealing due to its computational simplicity, it is a heuristic technique which can potentially yield a portfolio with both large risk and a large number of instruments.

In this paper, we model a combination of transaction cost and management cost as proportional to the units of instruments, yielding the problem

$$
\begin{array}{ll} 
& \min _{x \in \Re^{n}} \operatorname{risk}(x)+\alpha \sum_{i=1}^{n} c_{i}\left|x_{i}\right| \\
\text { subject to } & l_{x} \leq x \leq u_{x},
\end{array}
$$

where $c_{i}>0$ represents the cost for holding each unit of the $i$ th instrument and $\alpha \geq 0$ denotes the weight between minimizing the risk and cost. We note that, for a fixed $i, 1 \leq i \leq n$,

$$
\begin{array}{ll} 
& \min _{x \in \Re^{n}} \operatorname{risk}(x)+\alpha c_{i}\left|x_{i}\right| \\
\text { subject to } & l_{x} \leq x \leq u_{x},
\end{array}
$$

is the exact penalty formulation for the constrained problem

$$
\begin{array}{ll} 
& \min _{x \in \Re^{n}} \operatorname{risk}(x) \\
\text { subject to } & x_{i}=0, \\
& l_{x} \leq x \leq u_{x} .
\end{array}
$$

When the penalty parameter $\alpha c_{i} \geq 0$ is sufficiently large, the optimization problem (13) is equivalent to (14). Thus, $\alpha c_{i}$ represents the importance of excluding the $i$ th instrument in the hedging portfolio (in other words the cost of managing the $i$ th instrument). As we will illustrate subsequently, solving the risk minimization model (12) with different $c_{i}$ (various degree of cost consideration), one is able to achieve the objective of bounding the total number of replicating instruments (management cost).

We note that it is important to use the proportional cost function in order to account for management cost. If we consider the quadratic cost model,

$$
\begin{array}{ll} 
& \min _{x \in \Re^{n}} \operatorname{risk}(x)+\alpha c_{i} x_{i}^{2} \\
\text { subject to } & l_{x} \leq x \leq u_{x}
\end{array}
$$

(15) solves (14) only when $\alpha \cdot c_{i} \rightarrow+\infty$.

Next we use the same derivative portfolio example on the single stock to illustrate the properties of the optimal portfolios from the various models discussed. For a given hedging horizon $\tilde{t}>0$, we generate the future value matrix $\mathbf{V}$ from the prices $V_{i}\left(S_{\tilde{t}}, \tilde{t}\right)$ using Black-Scholes formula with 20000 independent samples of the stock price $S_{t}$ at $\tilde{t}$. Similarly, the future loss $\Pi^{0}\left(S_{\tilde{t}}, \tilde{t}\right)$ of the target portfolio is computed at the $S_{\tilde{t}}$ with the same parameters for appropriate analytic formulae. 
For all the computational results in this paper, $\left[x^{*}\right]_{\epsilon}$ denotes the total number of nonzero instruments of the portfolio $x^{*}$ calculated as the total number of instruments with $\left|x_{i}^{*}\right|>10^{-3}$, i.e., $\left[x^{*}\right]_{\epsilon} \stackrel{\text { def }}{=} \sum_{i=1}^{n} \zeta_{i}$ where $\zeta_{i}=1$ if $\left|x_{i}^{*}\right|>10^{-3}$ and $\zeta_{i}=0$ otherwise.

In order to facilitate a direct control on the hedge risk, instead of (12), we solve an equivalent problem

$$
\begin{array}{ll} 
& \min _{x \in \Re^{n}} \sum_{i=1}^{n} c_{i}\left|x_{i}\right| \\
\text { subject to } & l_{x} \leq x \leq u_{x}, \\
& \sqrt{\operatorname{risk}(x)} \leq u_{r},
\end{array}
$$

where $c_{i}$ can be interpreted as either the actual transaction cost per unit holding or the desirability of excluding the $i$ th instrument from the hedging portfolio. Here $u_{r}$ is an upper bound on the risk.

We compare the properties of the optimal hedging portfolios from the following three models:

- Model 0: the risk minimization model (7) with no bound constraint on the hedging position or cost consideration.

- Model 1: the risk minimization model (10) under the bound constraint:

$$
-\mathbf{E}\left(\Pi^{0}\right) \cdot /\left(V^{0}\right)^{T} \leq x \leq \mathbf{E}\left(\Pi^{0}\right) \cdot /\left(V_{0}\right)^{T} .
$$

Here, for $\beta>0, z \in \Re^{n}, \beta \cdot / z$ is a vector with the $i$ th component equal to $\beta / z_{i}$. This constraint specifies that the cost of each instrument cannot exceed the expected loss of the target portfolio.

- Model 2: the risk minimization model (16) under both a bound on the hedge risk and bound on the positions as in Model 1. For the computational results in this paper, we set

$$
u_{r}=\max \left((1+\rho) \sqrt{\operatorname{risk}\left(x_{m 1}^{*}\right)}, \rho\right)
$$

where $\rho>0$ is a constant and $x_{m 1}^{*}$ is the solution to Model 1 . When $u_{r}=(1+\rho) \sqrt{\operatorname{risk}\left(x_{m 1}^{*}\right)}$, $\rho$ specifies the accuracy of the risk compared to the optimal hedge risk obtained under only bound constraints.

To illustrate, we consider the same four different target portfolios examples. Table 2 compares the properties of the three hedging strategies from Model 0,1 , and 2 for a given time horizon of approximately three weeks with $\rho=0.5$. Here $\operatorname{risk}(0)=\frac{1}{m}\|b\|_{2}^{2}$, which corresponds to the risk when no hedging is performed, is also included for comparison. The following observations can be made from Table 2: First we observe that Model 1, with the specified bounds, has only a slightly larger risk compared to that of Model 0 , with the exception of the portfolio $P_{\mathrm{ba}}$ of barrier options. Note that the total number of the hedging instrument positions from Model 1 is much smaller due to the bound constraints. With $\rho=0.5$, the optimal risk achievable from Model 2 is slightly increased from that of Model 1. However, the number of instruments in the optimal portfolio has been decreased roughly $50 \%$. In addition, The initial hedging cost, cost $_{0}=V^{0} x^{*}$, of the optimal portfolios from all the three models are roughly the same (so are the cumulative hedging cost $\left.\Pi^{0}(S, \tilde{t})-V x^{*}+V^{0} x^{*}\right)$. This is reasonable since the optimal hedge risk is small and the initial cost of the hedging portfolio is roughly the expected future loss of the target portfolio.

Table 3 compares the properties of the optimal hedging portfolio from Model 2 but with $\rho=10$. We note that each optimal portfolio now has a smaller number of instruments compared to the corresponding portfolio with $\rho=0.5$; naturally the hedge risk is increased. 
Table 2: Comparisons of Optimal Portfolios: $\rho=0.5$

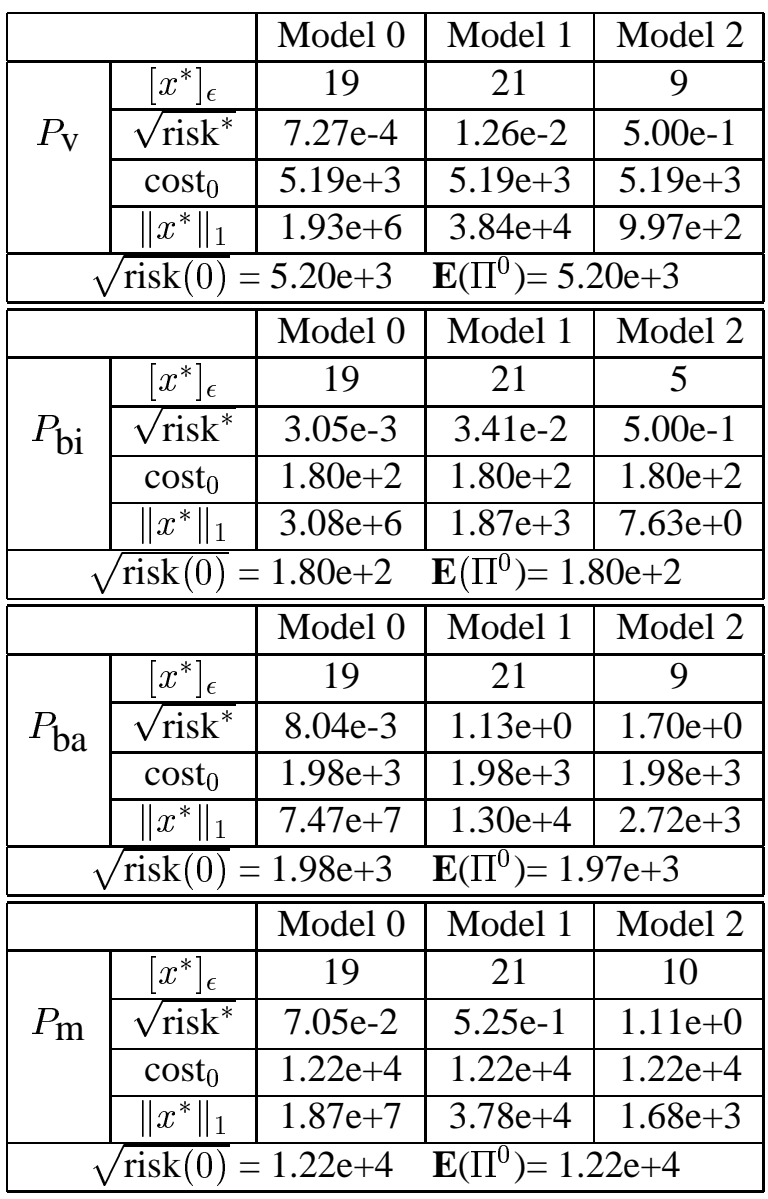

\section{Volatility Uncertainty}

In the previous section, we have illustrated that the risk minimization model (16) with cost consideration and bounds on the positions produces better hedging portfolios in that the instrument positions are smaller and there are fewer instruments in the optimal portfolio. The previous risk analysis examples, however, are based on the assumption that, at a future time $\tilde{t}>0$, the implied volatility is the same as the implied volatility at $t=0$. In practice, this is rarely the case; the future implied volatility is unobservable and uncertain.

In this section, we assume that the future derivative option values (and the loss of the target portfolio) depend on the future implied volatility $\sigma_{t}$, which is random, via the Black-Scholes formula (and the corresponding analytic formulae for the exotic options); let $V_{i}(S, \sigma, t)$ denote the value of an instrument at time $t$ when the underlying has value $S$ and implied volatility is $\sigma$. (The future interest rate and dividend yield are also uncertain but we ignore them in our discussion since their effect on option values is secondary comparing to that of the implied volatility.)

Assume that the change in the implied volatility is independently normally distributed with mean zero and a standard deviation of $\sigma_{\mathrm{vol}}$, i.e.,

$$
\sigma_{\tilde{t}} \in \mathcal{N}\left(\sigma_{0}, \sigma_{\text {vol }}\right)
$$

where $\sigma_{0}$ is the implied volatility at $t=0$ and $\mathcal{N}(\mu, \sigma)$ denote a normal distribution with mean of $\mu$ and standard deviation of $\sigma$. 
Table 3: Properties of Optimal Portfolios from Model 2: $\rho=10$

\begin{tabular}{|c|c|c|c|c|}
\hline & $P_{\mathrm{V}}$ & $P_{\mathrm{bi}}$ & $P_{\mathrm{ba}}$ & $P_{\mathrm{m}}$ \\
\hline$\left[x^{*}\right]_{\epsilon}$ & 6 & 2 & 6 & 7 \\
\hline$\sqrt{\text { risk }^{*}}$ & $1.00 \mathrm{e}+1$ & $1.00 \mathrm{e}+1$ & $1.25 \mathrm{e}+1$ & $1.00 \mathrm{e}+1$ \\
\hline cost $_{0}$ & $5.19 \mathrm{e}+3$ & $1.78 \mathrm{e}+2$ & $1.98 \mathrm{e}+3$ & $1.22 \mathrm{e}+4$ \\
\hline$\left\|x^{*}\right\|_{1}$ & $3.33 \mathrm{e}+2$ & $2.38 \mathrm{e}+0$ & $2.19 \mathrm{e}+2$ & $6.87 \mathrm{e}+2$ \\
\hline
\end{tabular}

Table 4: $\sqrt{\text { risk }}$ at $\tilde{t}$ When $\sigma_{\tilde{t}} \in \mathcal{N}(0.2,0.005): \rho=0.5$

\begin{tabular}{|c|c|c|c|}
\hline & Model 0 & Model 1 & Model 2 \\
\hline$P_{\mathrm{V}}$ & $6.28 \mathrm{e}+3$ & $3.38 \mathrm{e}+2$ & $3.46 \mathrm{e}+1$ \\
\hline$P_{\mathrm{bi}}$ & $1.46 \mathrm{e}+4$ & $8.54 \mathrm{e}+0$ & $5.84 \mathrm{e}-1$ \\
\hline$P_{\mathrm{ba}}$ & $1.73 \mathrm{e}+5$ & $5.75 \mathrm{e}+1$ & $7.57 \mathrm{e}+1$ \\
\hline$P_{\mathrm{m}}$ & $2.77 \mathrm{e}+5$ & $1.36 \mathrm{e}+2$ & $3.24 \mathrm{e}+1$ \\
\hline
\end{tabular}

For our previous examples, Model $0,1,2$ are solved with $\operatorname{risk}(x)=\frac{1}{m}\|\mathbf{V} x-b\|_{2}^{2}$ where $\mathbf{V}$ and $b$ are computed according to (8) and (9), assuming the implied volatility at timet is the same as the initial implied volatility. Table $4(\rho=0.5) \& 5(\rho=10)$ compare the risk produced by different models, when the future implied volatility has the distribution specified in (17) with $\sigma_{\mathrm{vol}}=.5 \%$. From column 2 in Table 4 , it can be seen that hedging using the optimal portfolio computed by the risk minimization Model 0 actually increases the risk significantly compared to simply not to hedge at all! It is surprising that such a small change of the implied volatility gives rise to such a large change in the risk; this large increase is due to the fact that the optimal risk minimization portfolio has large positions in the hedging instruments and this magnifies the model error significantly. From columns 3 and 4 in Table $4 \& 5$, the risk from the minimization with bound constraints (Model 1) and the minimization with bounds on both instrument positions and risk (Model 2) is much smaller than that of Model 0 . The results in Table 4 and 5 clearly illustrate that in hedging a portfolio, it is not necessary (or even not desirable) to minimize the hedging error due to the inevitable existence of model error. Rather it is important to limit the hedging error but also to consider other portfolio selection criteria, e.g., cost.

Table 4 and 5 illustrate that the risk of a hedging portfolio is very sensitive to the implied volatility; in fact the optimal hedging portfolios from Model 0 have increased the risk of the existing portfolio. In reality, just as it is impossible to know the actual future implied volatility, it is also impossible to know the exact model for the future implied volatility. Is it better to include a stochastic volatility model in risk minimization even though there is inevitable error in any assumed implied volatility model? We provide evidence below that the answer seems to be a definite yes.

We consider the same hedge risk minimization Model 0,1 , and 2 with $\operatorname{risk}(x)=\frac{1}{m}\|\mathbf{V} x-b\|_{2}^{2}$ except now the future values of the hedging instruments $\mathbf{V}$ are computed as below

$$
\mathbf{V}=\left[\begin{array}{ccc}
V_{1}\left(S^{1}, \sigma^{1}, t\right) & \cdots & V_{n}\left(S^{1}, \sigma^{1}, t\right) \\
\vdots & \cdots & \vdots \\
V_{1}\left(S^{m}, \sigma^{m}, t\right) & \cdots & V_{n}\left(S^{m}, \sigma^{m}, t\right)
\end{array}\right]
$$


Table 5: $\sqrt{\text { risk }}$ at $\tilde{t}$ When $\sigma_{\tilde{t}} \in \mathcal{N}(0.2,0.005): \rho=10$

\begin{tabular}{|c|c|c|c|}
\hline & Model 0 & Model 1 & Model 2 \\
\hline$P_{\mathrm{V}}$ & $6.28 \mathrm{e}+3$ & $3.38 \mathrm{e}+2$ & $2.41 \mathrm{e}+1$ \\
\hline$P_{\mathrm{bi}}$ & $1.46 \mathrm{e}+4$ & $8.54 \mathrm{e}+0$ & $1.00 \mathrm{e}+1$ \\
\hline$P_{\mathrm{ba}}$ & $1.73 \mathrm{e}+5$ & $5.75 \mathrm{e}+1$ & $2.61 \mathrm{e}+1$ \\
\hline$P_{\mathrm{m}}$ & $2.77 \mathrm{e}+5$ & $1.36 \mathrm{e}+2$ & $3.56 \mathrm{e}+1$ \\
\hline
\end{tabular}

and the loss of the target portfolio is given by

$$
b=\left[\begin{array}{c}
\Pi^{0}\left(S^{1}, \sigma^{1}, t\right) \\
\vdots \\
\Pi^{0}\left(S^{m}, \sigma^{m}, t\right)
\end{array}\right] .
$$

The condition number of $\mathbf{V}$ for a time horizon of a month is of the order of $10^{6}$ compared to $10^{16}$ when the implied volatility is assumed to be a constant. This is not surprising since the future values of the hedging instruments are affected by more risk factors: a delta-gamma approximation to the change $V_{i}(S, \sigma, t)-V_{i}\left(S_{0}, \sigma_{0}, 0\right)$ now has the form below:

$$
\begin{aligned}
& \left(\frac{\partial V_{i}}{\partial t}\right) \delta t+\left(\frac{\partial V_{i}}{\partial S}\right)^{T}(\delta S)+\frac{1}{2}\left(\frac{\partial^{2} V_{i}}{\partial S^{2}}\right)^{T}(\delta S)^{2} \\
& +\left(\frac{\partial V_{i}}{\partial \sigma}\right)^{T}(\delta \sigma)+\frac{1}{2}\left(\frac{\partial^{2} V_{i}}{\partial \sigma^{2}}\right)^{T}(\delta \sigma)^{2}+\left(\frac{\partial^{2} V_{i}}{\partial S \partial \sigma}\right)(\delta S)(\delta \sigma)
\end{aligned} .
$$

For our single asset derivative portfolio example, the risk minimization (2) has an infinite number of solution if $n>6$, instead of $n>3$, when the hedging instrument value is given by a delta-gamma approximation. Here we see that incorporating volatility risk adds more information in the risk minimization problem and improves the condition of the problem.

Table 6 displays the properties of the optimal portfolios from the risk minimization Model 0,1 , and 2 except now $\mathbf{V}$ and $b$ are computed from (18) and (19). We note that the optimal hedging portfolio without cost consideration has smaller holdings compared to the case when we assume that the implied volatility is a constant. T he optimal hedge risk is larger compared to when constant implied volatility is assumed in the risk minimization models, This is reasonable since it is harder to hedge away the additional volatility risk. In addition there seems to be a larger discrepancy between the initial cost and the expected future loss. Furthermore, Model 2 produces a hedging portfolio with fewer instruments, compared to that from Model 1 , with comparable hedge risks.

The optimal risk in Table 6 is computed with the assumption that the implied volatility $\sigma_{t}^{\circ}$ at time $\tilde{t}$ is normal distributed with a mean of $20 \%$ and a standard deviation of $.5 \%$; this model is likely to have error. Now suppose that the implied volatility at $\tilde{t}$ is deterministic and equal to the implied volatility at time $t=0$ (the same assumption made in the examples in the previous sections). What is the risk of th optimal portfolio computed with the assumption $\sigma_{\tilde{t}} \in \mathcal{N}(.20,0.005)$ ? Table 7 lists the risk for each model. We see that, relative to not hedging, significant risk has been reduced. In addition, the risk is also much smaller compared to the first column in Table $4 \& 5$ when the volatility uncertainty is not taken into consideration in risk minimization model but the implied volatility at $\tilde{t}$ is uncertain with a small standard deviation $\sigma_{\text {vol }}=.5 \%$.

In addition to Table 7, we have also investigated the situation when the implied volatility at $\tilde{t}$ has a slightly different distribution than what is assumed and found that incorporating volatility uncertainty leads to a more robust hedging strategy against model error (in implied volatility). In order to appreciate why 
Table 6: Properties of Optimal Portfolios: $\sigma_{\tilde{t}} \in \mathcal{N}(0.2,0.005)$ and $\rho=0.05$

\begin{tabular}{|c|c|c|c|c|}
\hline & & Model 0 & Model 1 & Model 2 \\
\hline \multirow{4}{*}{$P_{\mathrm{V}}$} & {$\left[x^{*}\right]_{\epsilon}$} & 21 & 21 & 14 \\
\hline & $\sqrt{\text { risk }^{*}}$ & $2.63 \mathrm{e}-1$ & $5.36 \mathrm{e}-1$ & $9.52 \mathrm{e}-1$ \\
\hline & cost $_{0}$ & $5.19 \mathrm{e}+3$ & $5.19 \mathrm{e}+3$ & $5.19 \mathrm{e}+3$ \\
\hline & $\left\|x^{*}\right\|_{1}$ & $1.29 \mathrm{e}+5$ & $2.22 \mathrm{e}+4$ & $6.81 \mathrm{e}+3$ \\
\hline \multicolumn{3}{|c|}{$\sqrt{\operatorname{risk}(0)}=5.20 \mathrm{e}+3$} & \multicolumn{2}{|c|}{$\mathbf{E}\left(\Pi^{0}\right)=5.20 \mathrm{e}+3$} \\
\hline & & Model 0 & Model 1 & Model 2 \\
\hline \multirow{4}{*}{$P_{\mathrm{bi}}$} & {$\left[x^{*}\right]_{\epsilon}$} & 21 & 21 & 12 \\
\hline & $\sqrt{\text { risk }^{*}}$ & $9.03 \mathrm{e}-2$ & $1.07 \mathrm{e}-1$ & $1.40 \mathrm{e}-1$ \\
\hline & $\operatorname{cost}_{0}$ & $1.80 \mathrm{e}+2$ & $1.80 \mathrm{e}+2$ & $1.80 \mathrm{e}+2$ \\
\hline & $\left\|x^{*}\right\|_{1}$ & $1.32 \mathrm{e}+4$ & $1.78 \mathrm{e}+3$ & $7.54 \mathrm{e}+1$ \\
\hline \multicolumn{3}{|c|}{$\sqrt{\operatorname{risk}(0)}=1.80 \mathrm{e}+2$} & \multicolumn{2}{|c|}{$\mathbf{E}\left(\Pi^{0}\right)=1.80 \mathrm{e}+2$} \\
\hline & & Model 0 & Model 1 & Model 2 \\
\hline \multirow{4}{*}{$P_{\mathrm{ba}}$} & {$\left[x^{*}\right]_{\epsilon}$} & 21 & 21 & 19 \\
\hline & $\sqrt{\text { risk }^{*}}$ & $9.93 \mathrm{e}-1$ & $2.83 e+0$ & $3.27 \mathrm{e}+0$ \\
\hline & $\operatorname{cost}_{0}$ & $1.98 \mathrm{e}+3$ & $1.98 \mathrm{e}+3$ & $1.98 \mathrm{e}+3$ \\
\hline & $\left\|x^{*}\right\|_{1}$ & $3.89 \mathrm{e}+5$ & $2.28 \mathrm{e}+4$ & $1.31 \mathrm{e}+4$ \\
\hline \multicolumn{3}{|c|}{$\sqrt{\operatorname{risk}(0)}=1.98 \mathrm{e}+3$} & \multicolumn{2}{|c|}{$\mathbf{E}\left(\Pi^{0}\right)=1.97 \mathrm{e}+3$} \\
\hline & & Model 0 & Model 1 & Model 2 \\
\hline \multirow{4}{*}{$P_{\mathrm{m}}$} & {$\left[x^{*}\right]_{\epsilon}$} & 21 & 21 & 8 \\
\hline & $\sqrt{\text { risk }^{*}}$ & $2.27 e+1$ & $2.27 \mathrm{e}+1$ & $2.38 \mathrm{e}+1$ \\
\hline & $\operatorname{cost}_{0}$ & $1.22 \mathrm{e}+4$ & $1.22 \mathrm{e}+4$ & $1.22 \mathrm{e}+4$ \\
\hline & $\left\|x^{*}\right\|_{1}$ & $9.09 \mathrm{e}+4$ & $7.02 \mathrm{e}+4$ & $1.21 \mathrm{e}+3$ \\
\hline & isk $(0)$ & $1.22 \mathrm{e}+4$ & \multicolumn{2}{|c|}{$\mathbf{E}\left(\Pi^{0}\right)=1.22 \mathrm{e}+4$} \\
\hline
\end{tabular}

this is so, we graph in Figure 1 the difference $\Pi^{*}(S, \sigma, \tilde{t})-\Pi^{0}(S, \sigma, \tilde{t})$ for the vanilla portfolio $P_{\mathrm{V}}$, where $\Pi^{*}(S, \sigma, \tilde{t})$ denotes the optimal hedging portfolio from Model 0 with $\mathbf{V}$ and $b$ computed from (8) and (9) (i.e., assuming the implied volatility $\left.\sigma_{\tilde{t}}=\sigma_{0}=.20\right)$. The difference $\Pi^{*}(S, \sigma, \tilde{t})-\Pi^{0}(S, \sigma, \tilde{t})$ with $\mathbf{V}$ and $b$ computed from (18) and (19), assuming (17) with $\sigma_{\mathrm{vol}}=.5 \%$, is graphed in Figure 2. Figure 1 shows that $\Pi^{*}(S, \sigma, \tilde{t})-\Pi^{0}(S, \sigma, \tilde{t})$ quickly becomes large when $\sigma$ deviates slightly from the initial implied volatility of $20 \%$ when the minimization model assumes that the implied volatility at $\tilde{t}$ is the same as the initial implied volatility (even though the time horizon $\tilde{t}$ is quite small). When the minimization model assumes that the future implied volatility has a slight error of .5\%, the hedging portfolio value is closer to the loss of the given portfolio for different implied volatilities. This results in a more robust hedge.

\section{Concluding Remarks}

In this paper, we illustrate why the problem of hedging a portfolio of derivatives is often ill posed. We propose to determine a hedging portfolio by minimizing a proportional cost subject to an upper bound on the hedge risk; this bound is typically slightly larger than the optimal hedge risk achievable without cost consideration. We illustrate that the optimal hedging portfolio obtained by the proposed method is more attractive since it consists of fewer instruments with a comparable risk. Finally we explain why including volatility uncertainty in hedge risk minimization results in a more robust hedging portfolio even though it is 
Table 7: $\sqrt{\text { risk }}$ at $\tilde{t}$ for Optimal Portfolios of Table 6 When $\sigma_{\tilde{t}}=\sigma_{0}=0.2$ and $\rho=0.05$

\begin{tabular}{|c|c|c|c|c|}
\hline & Model 0 & Model 1 & Model 2 & $\sqrt{\text { risk }(0)}$ \\
\hline$P_{\mathrm{V}}$ & $1.80 \mathrm{e}-1$ & $3.46 \mathrm{e}-1$ & $6.07 \mathrm{e}-1$ & $5.20 \mathrm{e}+3$ \\
\hline$P_{\mathrm{bi}}$ & $7.50 \mathrm{e}-2$ & $9.66 \mathrm{e}-2$ & $1.38 \mathrm{e}-1$ & $1.80 \mathrm{e}+2$ \\
\hline$P_{\mathrm{ba}}$ & $7.59 \mathrm{e}-1$ & $1.93 \mathrm{e}+0$ & $2.20 \mathrm{e}+0$ & $1.97 \mathrm{e}+3$ \\
\hline$P_{\mathrm{m}}$ & $1.36 \mathrm{e}+0$ & $1.36 \mathrm{e}+0$ & $4.06 \mathrm{e}+0$ & $1.22 \mathrm{e}+4$ \\
\hline
\end{tabular}

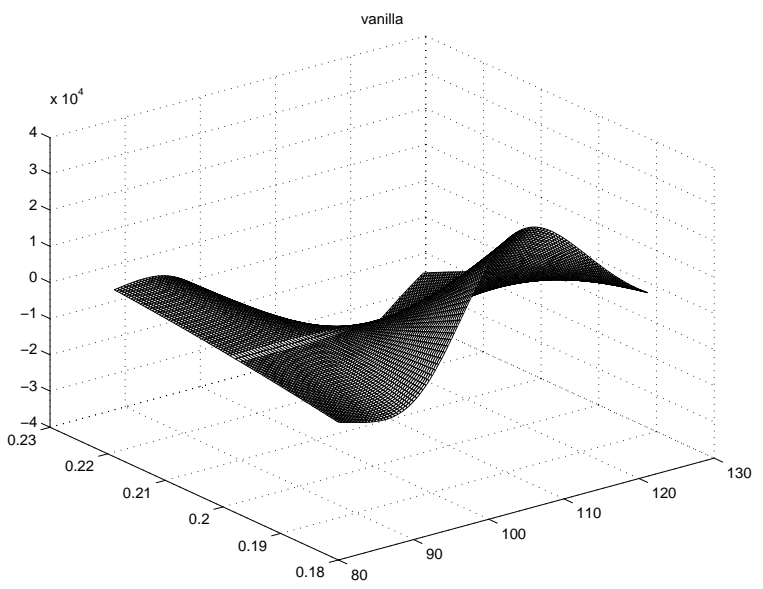

Figure 1: $\Pi^{*}(S, \sigma, \tilde{t})-\Pi^{0}(S, \sigma, \tilde{t})$ for $(S, \sigma) \in[80,130] \times[0.18,0.23]\left(\sigma_{\mathrm{vol}}=0\right.$ in Model 0$)$

impossible to know the exact model for the future implied volatility.

\section{References}

[1] S. Alexander, T. F. Coleman, and Y. Li. Minimizing VaR and CVaR. Technical report, Cornell University, January, 2003.

[2] Peter Carr and Liuren Wu. Static hedging of standard options. Technical report, Courant Institute, New York University, November 26, 2002.

[3] Thomas F. Coleman, Yuying Li, and M. Patron. Discrete hedging under piecewise linear risk minimization. Journal of Risk, to appear.

[4] S. Tompaidis. A new algorithm for hedging large portfolios of derivative instruments. In 12th Annual Derivatives Securities Conference. April 26-27, 2002, New York City. 


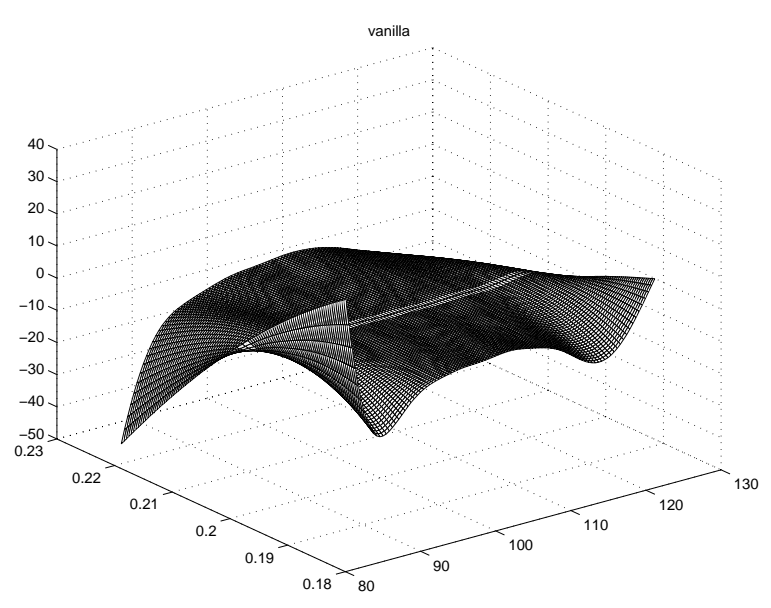

Figure 2: $\Pi^{*}(S, \sigma, \tilde{t})-\Pi^{0}(S, \sigma, \tilde{t})$ for $(S, \sigma) \in[80,130] \times[0.18,0.23]\left(\sigma_{\mathrm{vol}}=.5 \%\right.$ in Model 0$)$ 